\title{
Representaciones del Cáncer en Estudiantes de Medicina: Implicaciones para la Práctica y la Enseñanza
}

\author{
Representing cancer in undergraduate medical students: \\ implications for practice and teaching
}

\author{
Ana María Medina \\ Investigadora Independiente. ammedinach@unal.edu.co \\ Recibido 15 Diciembre 2006/Enviado para Modificación 10 Junio 2007/Aceptado 8 Agosto 2007
}

\section{RESUMEN}

Objetivo Como parte de un estudio a largo plazo el objetivo del presente estudio fue reconstruir las representaciones sociales de una parte del personal de salud estudiantes de medicina- en torno al cáncer y la manera como estas representaciones afectan la relación con los pacientes y familiares y su práctica médica.

Métodos La investigación integró métodos cualitativos propios de la psicología social y la antropología, en la educación médica. Para el desarrollo de esta fase se aplicaron cuatro grupos focales conformados por estudiantes de medicina, a lo largo de los años 2003 y 2004.

Resultados Para los estudiantes de medicina de primer semestre el cáncer se construye como un ente independiente de la persona que la padece y adquiere cualidades antropomórficas o zoomórficas, asociadas a la pérdida de límites y control. Los pacientes de cáncer son representados como personas sin futuro que pierden sus lazos sociales y afectivos.

Discusión En nuestro contexto es necesario pensar la posibilidad de cambio de las representaciones sociales, permitiendo nuevas maneras de relación con los pacientes de cáncer en los servicios médicos.

Palabras Clave: Cáncer, percepción social, educación de pregrado en medicina (fuente: DeCS, BIREME).

\section{ABSTRACT}

Objective This study was aimed at reconstructing social representations regarding cancer formed by a group of health personnel (medical students) as part of a longterm study and how such representations affect relationships with patients and family members and their medical practice.

Methods The investigation integrated qualitative methods from social psychology and anthropology in medical education. Four focal groups formed by medical 
students were applied throughout 2003 and 2004 for developing this phase. Results For first semester medical students cancer was seen as being an entity independent of the person being afflicted by it, acquiring anthropomorphic or zoomorphic qualities, associated with a loss of limits and control. Cancer patients were represented as being people without a future who would lose their social and affective links.

Discussion Social representations must become changed in our immediate setting, leading to new ways of relating to cancer patients in medical services.

Key Words: Cancer, undergraduate medical education, social perception (source: $\mathrm{MeSH}, \mathrm{NLM}$ ).

$\mathrm{E}$ n 1978 Susan Sontag -literata y activista de la libertad de expresiónescribió su libro "La enfermedad y sus metáforas" y años más tarde escribiría su complemento "El SIDA y sus metáforas". En los dos textos la autora planteaba cómo las metáforas y mitos que rodeaban ciertas enfermedades, especialmente el cáncer, añadían angustia y dolor emocional innecesario a los pacientes. Ella argumentó que el cáncer debía ser entendido como una enfermedad en tanto expresión biológica y sólo eso liberándolo de cualquier interpretación moral o social (1).

Su propuesta generó una buena cantidad de investigaciones que abordaron la temática. Existen por ejemplo algunos centrados sobre las representaciones del cáncer que tienen los pacientes o sus familiares en Brasil (2) y las implicaciones de las metáforas religiosas en la atención de un pacientes con cáncer (3); otros se centran en las representaciones que el personal de salud tiene de la enfermedad $(4,5)$ o su entrenamiento para el acompañamiento en el tratamiento del cáncer (6).

Sin embargo, no se encontraron estudios que aborden la educación médica y sus bases simbólicas. Es notorio que la mirada empirista clínica (7) que rige aún la aproximación a la enfermedad y al cuerpo humano en el contexto educativo colombiano deja de lado la aproximación a los niveles simbólicos de la enfermedad del propio personal de salud. La presunción de objetividad del personal de salud y de los datos recogidos a través de la observación en la mirada médica ha evitado exploraciones profundas sobre las representaciones sociales que los profesionales de salud tienen de la enfermedad y que hacen parte de su marco de referencia para la acción. La psicología, la sociología, la historia y la antropología han realizado aproximaciones críticas al concepto de salud y enfermedad, sin que impliquen una transformación estructural de los 
currículos universitarios ya que se enfrentan a una serie de obstáculos incorporados en las prácticas y los discursos (7).

Se presentan los resultados del análisis de cuatro grupos focales realizados con estudiantes de primer semestre de la carrera de medicina de una universidad privada de la ciudad de Bogotá, quienes realizaron un ejercicio reflexivo sobre sus representaciones de la enfermedad y quienes adicionalmente participaron de su análisis; con el objetivo de abordar niveles sociales y simbólicos del conocimiento que empezaban a adquirir en la carrera.

\section{MÉTODOS}

Participaron 50 estudiantes, divididos en grupos de 10 a 15 participantes, con los que se desarrollaron tres actividades: Primero realizaron una aproximación a sus propias representaciones sobre "la mujer" a través de un dibujo. Cada participante dibujó "una mujer" de acuerdo a lo que él o ella consideraran como tal. Posteriormente debían dibujar en otra hoja "el cáncer" y en una tercera hoja debían dibujar "una mujer con cáncer". El material fue analizado en grupo discutiendo las representaciones que emergían, los patrones de similitud y las diferencias. Posteriormente debían aplicar el taller a otras dos personas conocidas y discutir en grupo sus respuestas. El ejercicio tenía como objetivo fundamental ver qué tipo de representaciones individuales del cáncer surgían y generar un espacio de análisis y reconocimiento de las mismas como constructo social. El ejercicio tenía una clara orientación de género, en este caso particular enfocado en la representación de la mujer y la mujer enferma. El estudio se realizó entre el 2003 y 2004. Los dibujos se analizaron considerando algunos parámetros de análisis del interaccionismo: Actores presentes en la escena, escenario (en donde se ubican los actores), utilería (que objetos se incluyeron / excluyeron en el dibujo con el fin de significar escenas particulares), distribución espacial y uso dramático del escenario (manejo de espacio, color, luz, sombra, relaciones entre actores, presentación corporal) (8). Los parámetros de similitud eran clasificados de acuerdo a palabras claves descriptivas y a categorías emergentes propicias para el análisis y la reflexión.

\section{RESULTADOS}

Las representaciones sobre la mujer

Los hombres optaron por dibujar mujeres "atractivas y modernas", cuyas características centrales fueron: cabello largo, caderas anchas, sonrisa en la 
boca, peinadas, ropa que acentuara los senos y estómago. Se refieren a ella como "sin preocupaciones", "libre", "descomplicada", "feliz". La mujer dibujada es la mujer deseada. Las mujeres por su parte resaltaron que debían dibujar una mujer "bonita", completa, con una silueta marcada y esbelta, que debía estar a la moda y sin preocupaciones: es decir deseable. Todos incluyeron el cabello largo, los senos y las caderas como atributo femenino característico. Las mujeres fueron dibujadas casi en su totalidad de frente "para poderlas mirar a los ojos" y sonriendo, es decir dispuestas para la interacción. Se presume que están en el espacio público: bien vestidas, peinadas y arregladas. Algunas están acompañadas de libros y útiles escolares, mientras que otras posan para el artista (Figura 1).

Figura 1. Dibujos de "Mujer"

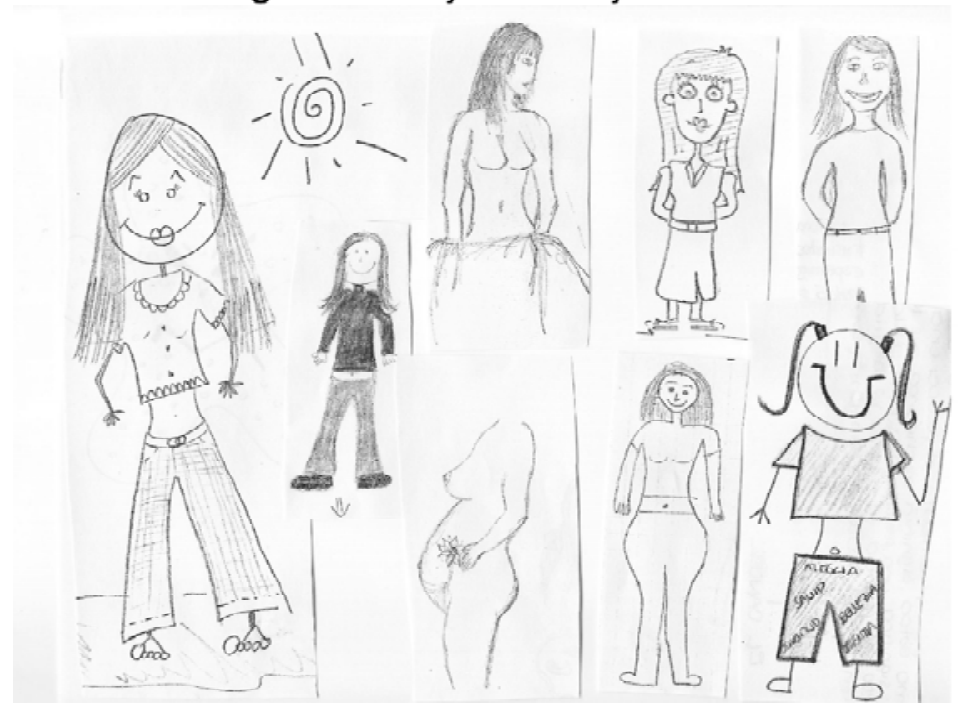

Las representaciones sobre el cáncer

El cáncer se representó como un ente vivo diferenciado del sujeto enfermo. Un ser con emociones humanas ubicado en lugares específicos del cuerpo, amorfo: "monstruo que poco a poco se va comiendo a la gente", un "ser oscuro con cachos, colmillos y pelos, de mal genio", "porque está buscando su próxima víctima". Otros estudiantes la representaron como una hoz junto a un reloj en aparente movimiento o un ángel de muerte sin rostro y sin manos; otros como una cruz en un cementerio, o una mano gigante que aplastaba al enfermo con un martillo. Dos optaron por utilizar el símbolo del cangrejo o el símbolo 
astrológico del mismo. Las palabras que acompañaron los dibujos fueron "invasión", "desesperanza", "muerte".

Se presentaron variaciones en la representación del cáncer en dos estudiantes que tenían un conocido con la enfermedad. En este caso el cáncer se representó como una $\mathrm{X}$ que cubría la boca "reflejando las mentiras que se decían", y otra joven describió el cáncer como la posibilidad de reflexión sobre la vida. Solo cinco estudiantes optaron por un dibujo dentro del modelo biológico de las células con una explicación sobre la patología en términos médicos. De este grupo, dos hicieron una representación intermedia, dibujando el esquema bidimensional básico de una célula pero tachado repetidas veces o rayado con fuerza (Figura 2).

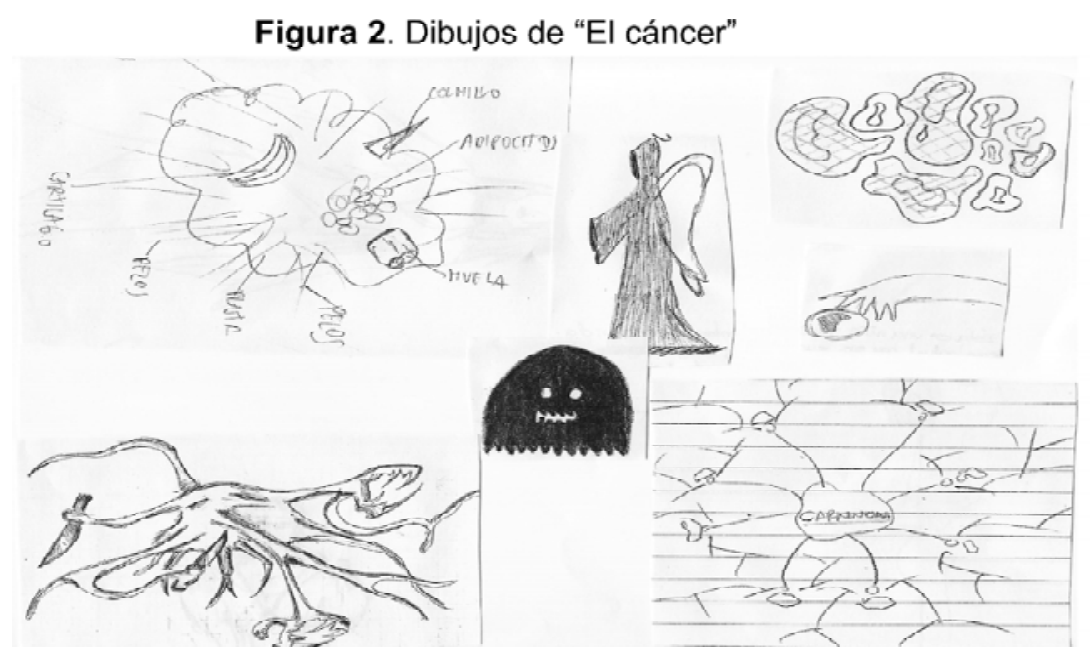

Las representaciones sobre la mujer con cáncer

Ante la solicitud de dibujar una mujer con cáncer, inmediatamente después de dibujar la "mujer" y el "cáncer" la respuesta fue: Desdibujar el límite del cuerpo dibujado con anterioridad rayándolo. Algunos borraron los límites. Otros tacharon el cuerpo o dibujaron figuras zoomórficas dentro del cuerpo de la mujer, como la conocida figura del juego electrónico PacMan devorando el interior de la mujer o produciendo un visible abultamiento. 
En general el escenario en que se dibujaron las mujeres fue de carácter hospitalario o en el cementerio. Su entorno se tornó oscuro y triste. La ropa cambia y el cuerpo deseable y deseado de la primera mujer es cubierto por ropas anchas y oscuras. Los ojos se entrecierran y en varios casos el cuerpo es postrado en una camilla acompañada de instrumental médico; pero en ningún caso se dibujaron así mismos como médicos de la paciente ni dibujaron ningún personal de salud o familiar alrededor: "la soledad" apareció también como palabra acompañante. De los cincuenta estudiantes 48 relacionaron el cáncer con cáncer de seno o ubicado en la zona reproductiva. Sólo dos lo ubicaron en una parte diferente del cuerpo: pulmón y piel. Las mujeres se representan tristes, supremamente delgadas, con el cabello rapado, los ojos hundidos y llorando. Las palabras que acompañan en general a los dibujos son "sufrimiento" y "dolor" (Figura 3).

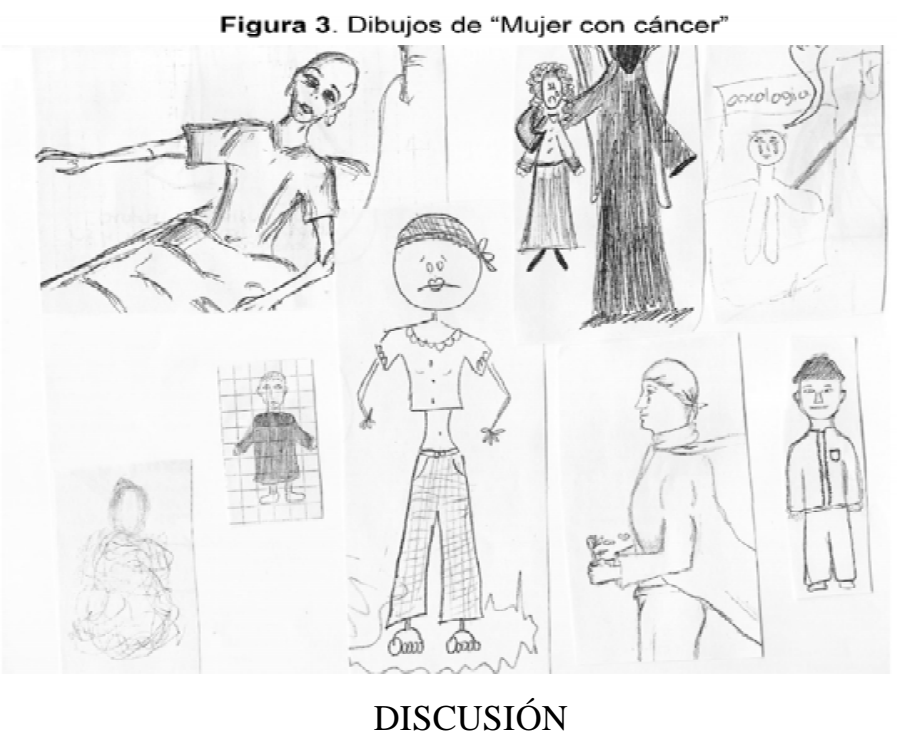

Dentro del discurso médico biologicista el cáncer es una de las pocas patologías que queda en una zona turbia para el conocimiento médico ya que no es fácilmente identificable a través de los síntomas o signos, no es completamente predecible y los tratamientos existentes no son completamente eficaces; y tal vez mas difícil aún, no existe una única causa identificada y por lo tanto tampoco su cura. Estas características hacen del cáncer una enfermedad propicia para las metáforas y los usos morales. En tanto desviación de la norma biológica el cáncer representa una gran ruptura del orden en el conocimiento y poder médico. 
Contrario a la opinión de Sontag, aquí se propone que es imposible eliminar las metáforas de la enfermedad o desmitificarlas ya que el pensamiento se constituye en un fuerte entramado de aspectos connotados y denotados de cualquier objeto o sujeto con el que los seres humanos se relacionen (9). Pensar en un conocimiento absolutamente denotado-objetivo-neutro y libre de interpretaciones o representaciones es prácticamente imposible: La vida familiar, las experiencias y eventos pasadas, las culturas particulares a las que se pertenezca, así como la información que circula en los medios masivos de comunicación constituyen los marcos de referencia -que se presentan sincrónicamente- para la acción en la vida cotidiana (10).

Por esto es importante que el estudiante de medicina, así como cualquier otro estudiante del área de la salud haga ejercicios en donde identifique no solo las raíces históricas del conocimiento médico que aprende, sino también en donde haga concientes las representaciones sociales que le servirán de marco de referencia subjetivo para la práctica médica. El estudiante debe reconocer en ellas una manera particular e histórica de percibir la enfermedad, así como una manera de posicionarse en el espacio social y adquirir poder. Es importante reconocer metáforas negativas como las encontradas otorgan mayor poder al sistema hospitalario que al paciente mismo; mientras las mujeres aparecen indefensas y solitarias enfrentando la enfermedad.

Al ubicar el cáncer en la zona pélvica y dorso se implica no solo la capacidad reproductiva sino la pérdida de la condición femenina deseable. En el cáncer de seno, la idea de un retiro parcial del seno, o de una cirugía reconstructiva no pasó por la mente de estos jóvenes, como tampoco la posibilidad de plantear otras alternativas terapéuticas fuera de la radio y quimioterapias. Este aspecto concuerda con una concepción de la enfermedad como una enfermedad local o regional y no de carácter sistémico o complejo, es decir que privilegia la mirada anatómica y ofrece como única salida la extirpación quirúrgica del órgano enfermo $(11,12)$. Cuando se identificaron causas del cáncer se asociaron a acciones individuales, como en el caso de culpar a la paciente por fumar (13). Aquí los datos epidemiológicos toman un curioso giro al convertir la probabilidad en una realidad individual que invisibiliza otras problemáticas y responsabilidades de tipo social y político como por ejemplo la calidad del aire de las grandes ciudades. El "mito de los estilos de vida" (7) ayuda en la prolongación de la idea hipocrática del régimen de vida general y de la responsabilidad individual sin reconocer la sujeción social del enfermo. 
Este tipo de representaciones centradas en el individuo y su responsabilidad así como en el miedo colectivo a la enfermedad son utilizados aún en las campañas de prevención y promoción en Colombia (14) y diariamente en la atención en las consultas individuales (15). Este tipo de representaciones negativas puede generar en la población reacciones que la lleven a ignorar o negar los mensajes o a que no vayan a los centros de atención (16).

Las instituciones educativas en áreas de la salud deben considerar la investigación y la educación entorno a estos campos del conocimiento, a partir de los cuales los profesionales de salud construyen una relación con la patología misma, con sus pacientes y con los familiares de los pacientes.

La antropología y la psicología social deben explorar mas profundamente el ámbito de la educación y la práctica clínica, proponiendo nuevos modelos de pensamiento sobre la enfermedad, que lleven necesariamente a la reconfiguración de las representaciones sociales de la misma y las metáforas que se utilizan para aprenderlas. Modelos que les permitan a los estudiantes adquirir nuevas herramientas simbólicas para relacionarse con sus pacientes, que les permitan plantear tratamientos integrales (17) o incluso que les ayuden al acompañamiento al bien morir (18). Es ya reconocido en el ámbito internacional del tratamiento oncológico que la metáfora es una poderosa herramienta para consolidar la relación terapéutica y un lenguaje común para aproximarse tanto a los discursos clínicos y los discursos del paciente de manera compartida (4). El futuro profesional debe tener la capacidad de construir un espacio dialógico, una trama lingüística y comunicativa accequible a las posibilidades interpretativas del paciente (9).

El estudiante de medicina debe adquirir las herramientas para identificar qué tipo de metáforas son positivas o culturalmente coherentes para las personas que va a atender. Para algunos pacientes las metáforas de guerra o de monstruos devoradores puede ser positiva ya que estás imágenes le permiten constituir una identidad nueva de "luchador". Sin embargo tienen una carga masculina y agresiva que puede no ser adecuada para otros/as pacientes. Pueden ser por el contrario absolutamente inhibidores de acción frente a la enfermedad, asignando culpas innecesarias al paciente si no puede cumplir con las expectativas de la lucha. Identificar los núcleos de significado de las metáforas y sus interrelaciones con la vida del paciente puede ser muy útil al acompañar el padecimiento o en la conformación de grupos de autoayuda $(19,20)$. El estudiante de medicina debe aprender a aceptar diversas formas de representar la enfermedad que le permitan interactuar con pacientes diferentes y actuar 
sobre la enfermedad y el padecimiento. Igualmente la capacidad para identificar y analizar sus propias representaciones le ayudará en un futuro a explorar sus usos en contextos organizacionales o institucionales (21).

Una aproximación al inconciente intelectual y simbólico de los profesionales de salud, así como su uso en las campañas de promoción y prevención, en especial el uso de la metáfora, puede ser una valiosa herramienta analítica que permita aproximarse a niveles intersubjetivos que enmarcan el conocimiento y las prácticas en su interior (22). Este tipo de investigaciones, sin embargo debe mantener su condición crítica: Es decir no desligar las representaciones sociales de una realidad social, económica y política, así como del uso moral que las metáforas condensan (23). Una posición intermedia entre las investigaciones centradas en el discurso y aquellas centradas en las condiciones materiales y de producción puede ser útil para comprender y plantear las reformas que la educación médica y en salud requiere.

Los contenidos de género deben ser cuidadosamente analizados en los discursos médicos (tanto en la práctica como en la educación médica) ya que a partir de ellos se puede ayudar a construir realidades simbólicas que impidan o limiten a los pacientes que no se identifican con dichas realidades o que sienten excluidas partes importantes de su vida social y afectiva.

La representación de las enfermedades ubica socialmente tanto al médico como al paciente y por lo general este posicionamiento implica una relación inequitativa entre quienes poseen el conocimiento y quienes no, entre hombres y mujeres y entre diferentes tipos de profesionales de salud (23). Es importante que el estudiante identifique críticamente sus propias representaciones y la manera cómo estas le sirven de marco de referencia y acción para la vida cotidiana. La aproximación conciente a las representaciones permitirá comprender mejor la complejidad de los servicios de salud, así como la generación de nuevas metáforas que permitan modificaciones en los espacios de trabajo en salud \&

\section{REFERENCIAS}

1. Sontag S. La enfermedad y sus metáforas y el sida y sus metáforas. Madrid: Taurus; 1996.

2. Tavares JSC, Trad LAB. Breast cancer metaphors and meanings from the perspective of five families. Cad. Saúde Pública, 2005; 21:426-435 
3. Astrow A, Mattson I, Ponet R, White M. Inter-Religious perspectives on hope and limits in cancer treatment. JClin Oncol, 2005; 23(11): 2569-2573.

4. Reisfield, GM, Wilson, GR.Use of metaphor in the discourse on cancer. J Clin Oncol, 2004; 22(19): 4024-4027.

5. Arroliga A, Newman S, Longworth DL, Stoller JK. Metaphorical medicine: Using metaphors to enhance communication with patients who have pulmonary disease. Ann Intern Med, 2002; 137(5_Part_1): 376-379.

6. Penson R, Schapira L, Daniels KJ, Chabner BA, Lynch, TJJr. Cancer as metaphor. Oncologist, 2004. 9(6): 708-716.

7. Quevedo E. El proceso de salud enfermedad: Hacia una clínica y una epidemiología no positivistas. En: A. Cardona, Ed. Sociedad y Salud. Bogotá: Zeus Editores, 1992.

8. Goffman E. La presentación de la persona en la vida cotidiana. Buenos Aires: Amorrortu; 1971.

9. Restrepo LC, Espinel M. Enfermedad y significación. Revista Debates en Psicología, 1997; 1:97-110

10. Modena ME. Combinar recursos curativos: un pueblo mexicano en las últimas décadas del siglo XX. En: Armus D, Editor. Entremédicos y curanderos: Cultura, historia y enfermedad en la América Latina moderna. Buenos Aires: Grupo Editorial Norma; 2002.

11. Lerner BH. Fighting the War on Breast Cancer: Debates over early detection, 1945 to the Present. Ann Intern Med 1998; 129(1):74-78.

12. Foster RSJr. Breast cancer detection and treatment: A personal and historical perspective. Arch Surg 2003; 138(4):397-408.

13. Chapple A, Ziebland S, McPherson, A. Stigma, shame, and blame experienced by patients with lung cancer: qualitative study. BMJ 2004; 328:1470.

14. Medina AM, Jaime S. Mensajes sin prevención, estudio del caso de las vallas sobre el cáncer en Bogotá en el 2002. Memorias I Congreso Nacional de Investigación en Salud Pública. Bogotá, Pontificia Universidad Javeriana; 2006.

15. Press N, Fishman JR, Koenig BA. Collective fear, individualized risk: the social and cultural context of genetic testing forbreast cancer. Nursing Ethics 2000; 7(3): 237-249.

16. Ray M, Wilkie W. Fear: The potential of an appeal neglected by marketing. Journal of Marketing 1970; 34(1): 54-62.

17. Stine JJ. The use of metaphors in the service of the therapeutic alliance and therapeutic communication. Journal of the American Academy Of Psychoanalysis And Dynamic Psychiatry 2005; 33(3): 531-545.

18. Hutchings D. Communicating with metaphor: A dance with many veils. American Journal of Hospice and Palliative Medicine 1998; 15(5): 282-284.

19. Coreil J, Wilke J, Pintado I. Cultural models of illness and recovery in breast cancer support groups. Qual Health Res 2004; 14(7): 905-923. 
20. Colbourne L, Sque M. The culture of cancer and the therapeutic impact of qualitative research interviews. Journal of Research in Nursing 2005; 10(5): 551-567.

21. McCrickerd J. Metaphors, models and organisational ethics in health care. J. Med. Ethics 2000; 26(5): 340-345.

22. Aita V, McIlvain H, Susman J, Crabtree B. Using metaphor as a qualitative analytic approach to understand complexity in primary care research. Qual Health Res 2003; 13(10): 1419-1431.

23. Bishop FL, Yardley L. Constructing agency in treatment decisions: Negotiating responsibilityin cancer. Health 2004; 8(4): 465-482. 